maladies infectieuses : destinée fatale des enfants nourris artificiellement.

En considérant le problème de ce point de vue, il n'est personne qui ne voit la grande importance que prennent ces études de chimie colloïdale dans la pratique pédiatrique ; études que, du reste, nous entendons poursuivre de plus en plus, dans le but d'éclaircir également certains points restés jusqu'à présent obscurs du problème de la nutrition infantile.

\title{
RÉACTION PRESQUE AUSSI RAPIDE ET FACILE QUE ZLA RÉACTION DE STORCH POUR DISTINGUER DU LAIT CRU UN LAIT DE PASTEURISATION BASSE OU UN LAIT STASSANISÉ
}

\author{
par M. C. C. Christen, Ingénieur-Chimiste
}

en mission du Gouvernement de Santa Fé (République Argentine)

On sait que le lait de pasteurisation basse, par le fait même d'être moins touché par le chauffage que le lait de pasteurisation haute, accuse encore la présence en assez bon état de conservation de la peroxydase. Il s'ensuit qu'il n'est pas possible de le distinguer du lait cru par la réaction à la paraphénylènediamine, couramment dite réaction de Storch, pas plus que par les autres réactions similaires de laboratoire encore plus délicates et constantes, notamment celle à l'eau saturée de gayacol en présence de traces d'eau oxygénée (Réaction de Dupouy, antérieure d'ailleurs à la réaction de Storch).

$\mathrm{Au}$ fond, la persistance de ce ferment soluble dont nous ignorons encore la signification et l'origine, dans un lait après la pasteurisation, est à l'honneur du procédé employé au lieu d'en être considéré comme un défaut. On serait en effet porté à croire qu'il en est plutôt un défaut si l'on prêtait attention aux difficultés que certains chimistes, officiels ou non, très attachés aux vieilles pratiques, soulèvent lorsqu'on leur parle d'unsystème nouveau de pasteurisation qui respecte la peroxydase. Avec effarement ils disent : " $\mathrm{Oh}$, la réaction de Storch est négative. "

Voici une réaction presque aussi facile et rapide que celle de Storch, et qui comme cette dernière est parfaitement à la portée des petits laboratoires. Les deux procédés que M. le Professeur Orla-Jensen (Le Lait, juin 1929) vient de proposer offrent certainement de beaucoup plus grandes difficultés pour entrer dans la pratique courante

A de gros tubes à essai, renfermant chacun environ $30 \mathrm{~cm}^{3}$ de lait écrémé additionné de tournesol, le tout parfaitement stérile, on ajoute, à l'aide d'une pipette également stérile et avee les soins habituels d'asepsie, un échantillon du lait en examen: soit $3 \mathrm{~cm}^{3}$, soit $10 \mathrm{~cm}^{3}$. 
Le mélange ne change pas sensiblement de couleur, la teinte caractéristique bleu, tirant légèrement au violet, du tournesol, persiste, aussi bien après l'addition de $10 \mathrm{~cm}^{3}$ que de $3 \mathrm{~cm}^{3}$ de lait.

On porte le tube à l'étuve à $37^{\circ}$ ou à $40^{\circ}$ ou dans un simple bainmarie. L'essai réussit encore mieux à une température supérieure pourvu que l'on ne dépasse pas $45^{\circ}$.

En moins de 3 heures, si le lait additionné est du lait cru, le mélange devient absolument blane; la décoloration est plus rapide, et survient au bout de 1 1/2 heure environ après l'addition de $10 \mathrm{~cm}^{3}$.

$\mathrm{Au}$ contraire, si le lait additionné est du lait de pasteurisation basse ou du lait stassanisé, la décoloration du mélange est très tardive. Elle débute seulement après 6 heures.

Cette réaction, à vitesses si différentes dans les deux cas, est due parallèlement à l'acidification du lait et à la réduction de la substance colorante du tournesol.

Il y a done à la fois virage et décoloration. Lentement cependant, au contact de l'air, la leucobase du tournesol reprend son oxygène, se parant ainsi de la nuance correspondante au degré d'acidité atteint à ce moment par le lait.

On peut contrôler cette réaction par l'essai suivant également à la portée des laboratoires de vérification des denrées alimentaires : Le lait à examiner. distribué dans des petites fioles stériles, est porté tel que à l'étuve à $40^{\circ}$ et même plus haut, mais sans dépasser $45^{\circ}$.

$\mathrm{Au}$ bout du même laps de temps, 3 heures, si l'échantillon soumis à l'épreuve est du lait cru, son acidité initiale monte de 7 à $8^{\circ} \mathrm{S}$. et même davantage. S'il s'agit, par contre, de lait de pasteurisation basse, cette montée sera très réduite et ne dépassera pas plus de $0^{0} 7$ à $0^{\circ} 8 \mathrm{~S}$. Avec le lait stassanisé, pareil écart sera encore plus réduit ; il sera de $0^{\circ} 2 \mathrm{~S}$, au plus.

Dans ce second essai, la différence est aussi nette que dans le précédent.

Nous extrayons de notre carnet de notes quelques-uns des résultats que nous avons obtenus en opérant comparativement sur le lait cru et du même lait traité simultanément par le procédé de la pasteurisation basse (P. B.) et par le procédé de la couche mince (Stassaniastion : (S.) :

Etuve à $37^{\circ}$ (acidité en degrés Soxhlet.)

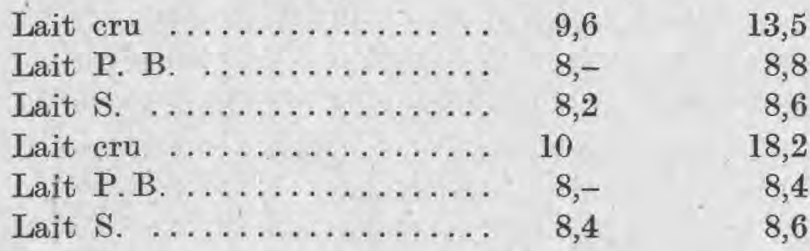




\section{Etuve à $40-43^{\circ}$ centigrades}

\begin{tabular}{|c|c|c|}
\hline Lait cru & 8,6 & 19,4 \\
\hline Lait P. B. . . . . . . . . . & 8,2 & 8,8 \\
\hline Lait $\mathrm{S} . \ldots \ldots \ldots \ldots \ldots \ldots$ & $8,-$ & 8,2 \\
\hline Lait e & 8,4 & 17,6 \\
\hline Lait P. B............... & 8,2 & \\
\hline Lait $\mathrm{S} . \ldots \ldots \ldots \ldots \ldots \ldots \ldots$ & $8,-$ & \\
\hline
\end{tabular}

Note: Les tubes de lait écrémé au tournesol sont d'un usage courant à l'Institut Pasteur. Leur introduction dans la pratique semble due à Metchnikoff qui s'en servait, en tout cas, dans ses recherches sur les ferments lactiques.

La teinture de tournesol est préparée en délayant 125 grammes de poudre de tournesol dans un litre d'eau distillée. La liqueur est filtrée puis stérilisée par tyndallisation 3 fois à $105^{\circ}$.

On emploie du lait écrémé très frais, on y ajoute la teinture stérilisée au tournesol dans la proportion du $10 \%$.

On distribue dans des gros tubes à essai, $30 \mathrm{~cm}^{3}$ environ par tube, et on stérilise à l'autoclave pendant 20 minutes à $115^{\circ}$.

\section{(Travail du Laboratoire de la Laiterie Centrale de Strasbourg exécuté sous la direction du Docteur Stassano.)}

\section{Note de M. Henri STASSANO}

Après le départ de $\mathrm{M}$. Christen, j'ai pu constater qu'il y a avantage, en tant que rapidité d'action et simplification du procédé, à remplacer les tubes de lait stérilisé additionné de tournesol par la simple addition au lait en examen de quelques gouttes de bleu méthylène. $\mathrm{Au}$ fond, la réaction en question est la réaction de la réductase. Helmoltz, le premier en 1844, a vait remarqué la réduction du tournesol par les bactéries, mais c'est indiscutablement sur le bleu de méthylène que la production de la leuco-base, le blanchissement, apparaît plus ra pidement,

Il y a néanmoins şrand intérêt a pratiquer simultanément avec l'épreuve au bleu de métbylène (réductase), l'épreuve de l'augmentation de l'acidité en réci pient stérile à l'étuve à $40^{\circ}$.

D'après les déterminations de ce genre qui sont pratiquées à présenten série à la Laiterie Centrale sur un grand nombre d'échantillons de laits crus. même le laps de temps de 2 heures suftit pour que l'on $\mathrm{y}$ constate parfois des augmentations de 10 à 11 degrès Soxhlet. 